\title{
乌s \\ Compression of a photoinjector electron bunch in the negative-mass undulator
}

\author{
Ilya V. Bandurkin, ${ }^{1}$ Ilya S. Kurakin, ${ }^{2}$ and Andrey V. Savilov ${ }^{1}$ \\ ${ }^{1}$ Institute of Applied Physics, Nizhny Novgorod, 603950, Russian Federation \\ ${ }^{2}$ Lobachevsky Sate University of Nizhny Novgorod, 603950, Russian Federation
}

(Received 9 November 2016; published 15 February 2017)

\begin{abstract}
The use of the "negative mass" regime provides stabilization of longitudinal size of dense photoinjector electron bunches moving through a long undulator. This allows one to increase significantly the power capabilities of a terahertz source based on coherent spontaneous emission from a short bunch. However, such type of emission is produced if the bunch length is comparable with the radiation wavelength. This work discusses the use of the negative mass regime to provide effective compression of dense bunches down to "terahertz" lengths.
\end{abstract}

DOI: 10.1103/PhysRevAccelBeams.20.020704

\section{INTRODUCTION}

Laser-driven photoinjectors allow formation of fairly compact and accessible sources of dense electron bunches with a moderate energy of 3-6 MeV, picosecond pulse durations, and charges of up to $1 \mathrm{nC}$ and even greater [1-5]. These bunches can be directly exploited for radiation in the $\mathrm{THz}$ frequency range [6-13]. They can be used, in particular, for realization of comparatively simple and compact sources operating in the regime of spontaneous coherent undulator radiation. This type of radiation is realized when the effective axial length of bunches in the radiation section is shorter than the wavelength. In this situation, the wave packets emitted by each of the electrons add up basically in phase.

Evidently, the length of the operating region of such a source is strictly limited by the Coulomb repulsion leading to an increase in the axial size of the bunch. The process of spontaneous coherent radiation stops when the bunch becomes longer than the radiation wavelength. Paper [12] describes a method of significant weakening the axial repulsion and, simultaneously, of confining particles in the axial direction by means of using the undulator with strong axial uniform magnetic field. In this system, the "negative-mass" effect occurs when the electron cyclotron frequency corresponding to the guiding magnetic field exceeds the bounce frequency of oscillations in the periodic undulator field [14-16]. The term "negative mass" denotes a situation, when an increase/decrease in the kinetic energy of the particle [determined by the relativistic Lorents factor, $\gamma=\left(1-v^{2} / c^{2}\right)^{-1 / 2}$ ] leads to the opposite change

Published by the American Physical Society under the terms of the Creative Commons Attribution 4.0 International license. Further distribution of this work must maintain attribution to the author(s) and the published article's title, journal citation, and DOI. (decrease/increase, respectively) in its axial velocity, $v_{z}$, so that $\delta v_{z} / \delta \gamma<0$. Axial Coulomb repulsion results in acceleration of electrons moving in the front of the bunch and in deceleration of particles moving in the tail (Fig. 1). Since the change in the axial velocity is opposite to the change in energy in the negative-mass regime, the tail of the bunch moves faster than the head. Thus, the Coulomb interaction between the electrons leads to their mutual attraction. This is very similar to the negative-mass effect in electron beams used in cyclotron masers [17-21]. It is shown in works $[12,13]$ that the use of the negative-mass stabilization of an electron bunch provides significant power enhancement and spectrum narrowing for a $\mathrm{THz}$ radiation source based on the spontaneous coherent undulator emission from a short bunch.

Naturally, the spontaneous coherent character of emission is provided, if the length of the bunch is comparable (at least) with the radiation wavelength. However, achieving "terahertz" ( $0.3 \mathrm{~mm}$ and shorter) lengths of electron bunches at a high density (and high Coulomb fields) is a complicated problem. On the other hand, since the use of the negative-mass regime solves the problem of the Coulomb repulsion, it is natural to use such a regime to provide effective compression of a relatively long electron bunch down to terahertz lengths. In this case, the rf source based on the spontaneous coherent undulator emission from a short bunch should include two negative-mass undulators (Fig. 1). The first one plays the role of a compressor of the electron bunch. In this undulator, the effective axial mass of electrons is negative and small, so that $\delta v_{z} / \delta \gamma<0$ and $v_{z}$ change fast enough with the change in $\gamma$. In this situation, the Coulomb attraction of electrons is strong, and the axial size of the bunch decreases rapidly in the process of the motion of the bunch through this undulator. In the point of the minimal size, the undulator period is changed to provide the regime of an "infinitely high axial mass" of electrons. This means that the effective 


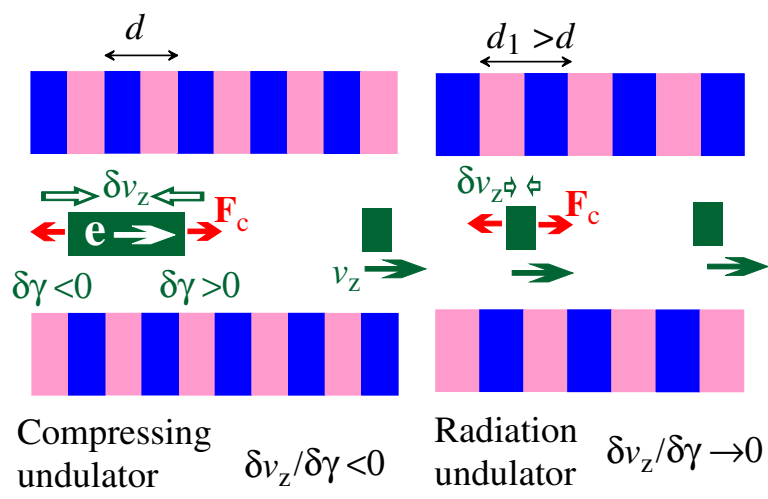

FIG. 1. Schematic of a rf source based on spontaneous coherent emission from short electron bunches, which includes compression and radiation sections.

axial mass of electrons stays negative $\left(\delta v_{z} / \delta \gamma<0\right)$, but the change in electron energy results in a very slow change in the axial electron velocity $\left(\left|\delta v_{z} / \delta \gamma\right| \rightarrow 0\right)$. In this regime, the Coulomb attraction of electrons is very weak, and the axial bunch size is approximately stabilized. Therefore, the second section operates as a radiator providing a long-length process of the spontaneous coherent undulator emission from a short and stable bunch.

Naturally, the negative-mass compression of electron bunches may be of value in itself, then there is no further use for realization of a $\mathrm{THz}$ source based on spontaneous coherent emission from compressed bunches. Therefore, the subject of this paper is limited to the consideration only of the process of the negative-mass compression. Section II describes a simplified model of a short electron bunch used in this consideration. In Sec. III, a simple two-particle approximation is used to illustrate the behavior of an electron bunch moving through the negative-mass undulator by means of the quasianalytical phase-plane approach. Several examples of numerical simulations of the motion of an electron bunch through the negative-mass undulator are given in Sec. IV.

\section{MODEL OF A SHORT ELECTRON BUNCH MOVING IN THE NEGATIVE-MASS UNDULATOR}

We consider the motion of particles of a short dense bunch along a helically polarized undulator with a strong guiding magnetic field [Fig. 2(a)]. In the relativistic equation of motion,

$$
\frac{d \mathbf{P}}{d t}=-\frac{e}{c} \mathbf{v} \times\left(\mathbf{B}_{u}+\mathbf{B}_{0}\right)-e \mathbf{E},
$$

we take into account three kinds of fields. First, the periodic magnetic field of the helically polarized undulator is described by the following formula:

$$
\mathbf{B}_{u}=B_{u}\left[\mathbf{x}_{0} \cos (h z)+\mathbf{y}_{0} \sin (h z)\right] .
$$

Here, we neglect transverse inhomogeneity of the undulator field in the scale of the transverse cross section of the electron bunch. Second, there exists a uniform guiding magnetic field:

$$
\mathbf{B}_{0}=B_{0} \mathbf{z}_{0}
$$

Finally, electric field $\mathbf{E}$ in Eq. (1) describes the mutual Coulomb interaction of particles in the electron bunch. We take into account only axial Coulomb interaction between electrons, $\mathbf{E}=z_{0} E$, having assumed that the Coulomb distraction of electrons in the transverse direction is compensated by the magnetic interaction of the relativistic particles, as well as by the presence of the axial magnetic field and focusing action of the undulator.

In order to calculate the axial electric field inside the bunch, we represent the bunch as a discrete set of $N$ fractions [thin "pancakes," Fig. 2(b)]; each of them has the electric charge $q=Q / N$, where $Q$ is the total charge of the bunch. In the reference system related with the electron bunch, the axial electric field of the jth "pancake" at the axis of the symmetry [Fig. 2(c)] is determined by the following formula:

$E\left(z^{\prime}-z_{j}^{\prime}\right)=\frac{-2 \pi q}{S} \times \operatorname{sign}\left(z^{\prime}-z_{j}^{\prime}\right) \times\left[1-\frac{1}{\sqrt{1+R^{2} /\left(z^{\prime}-z_{j}^{\prime}\right)^{2}}}\right]$.

Here, $z^{\prime}$ is the axial coordinate in the corelated system, $z_{j}^{\prime}$ is the coordinate of the jth pancake, $R$ is the radius of the electron bunch, and $S=\pi R^{2}$ is the area of the cross section of the bunch. We assume that the same electric field acts to all particles of the ith pancake. Then, the field acting from the jth pancake to the ith pancake is determined by the following formula:
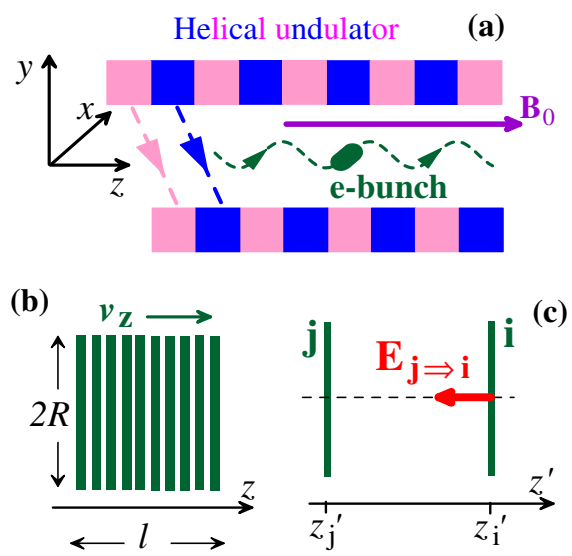

FIG. 2. (a) Electron bunch moving in a helical undulator with guiding magnetic field. (b) The model of a bunch in the form of a set of charged pancakes. (c) Electric field acting from the jth pancake to the ith one. 


$$
E_{j \rightarrow i}=\frac{-2 \pi}{S} \times \frac{Q}{N} \operatorname{sign}\left(z_{i}-z_{j}\right) \times\left[1-\frac{1}{\sqrt{1+R^{2} / \gamma_{j}^{2}\left(z_{i}-z_{j}\right)^{2}}}\right] .
$$

Here, $\gamma$ is the relativistic electron Lorentz factor, and the Lorentz reduction of the axial coordinate is taken into account. Thus, the total electric field acting to the ith pancake is found as a sum over all pancakes:

$$
\begin{aligned}
E_{i}= & \sum_{j=1}^{N} E_{j \rightarrow i} \\
= & \frac{-2 \pi Q}{S} \times \frac{1}{N} \sum_{j=1}^{N} \operatorname{sign}\left(z_{i}-z_{j}\right) \\
& \times\left[1-\frac{1}{\sqrt{1+R^{2} / \gamma_{j}^{2}\left(z_{i}-z_{j}\right)^{2}}}\right] .
\end{aligned}
$$

For illustration, let us estimate the rate of expanding of the electron bunch in the case, when the undulator is absent. In this case, electron motion has only axial character,

$$
m \gamma^{3} \frac{d v_{z}}{d t}=-e E
$$

and the current length of the bunch (the distance between the two particles moving in the front and in the tail of the bunch) is described as follows:

$$
m \gamma^{3} \frac{d^{2} l}{d t^{2}}=2 e\left|E_{0}\right|,
$$

where $\left|E_{0}\right|$ is the absolute value of the electric field at the edges of the bunch. According to Eq. (3), if the bunch is short enough $(\gamma l \ll R)$, then this field can be approximated as the field of an infinite charged plane, $\left|E_{0}\right| \approx 2 \pi Q / S$. This leads to the following estimation for the ratio between the current length of the bunch and its initial length:

$$
\frac{\delta l}{l_{0}} \approx \frac{z^{2}}{R^{2}} \gamma^{-3} \frac{2 I}{I_{A}} .
$$

Here, $\delta l=l-l_{0}$ is the change in the bunch length, $z=v t \approx c t$ is the current axial coordinate of the bunch, $I \approx c Q / l_{0}$ is the initial electron current in the bunch, and $I_{A}=m c^{3} / e \approx 17 \mathrm{kA}$ is the Alfven current.

As an example, let us consider $0.5 \mathrm{nC} / 1 \mathrm{ps}$ electron bunch $\left(I=500 \mathrm{~A}\right.$ and $\left.l_{0}=0.3 \mathrm{~mm}\right)$ with a radius of $R=1 \mathrm{~mm}$, which is accelerated up to an energy of $5 \mathrm{MeV}(\gamma \approx 11)$. In this situation, the electron bunch becomes twice as long $\left(\delta l / l_{0} \approx 1\right)$ at the distance $z \approx 15 \mathrm{~cm}$, and it becomes 3 times as long $\left(\delta l / l_{0} \approx 2\right)$ at the distance $z \approx 20 \mathrm{~cm}$.

\section{ILLUSTRATION OF THE NEGATIVE-MASS EFFECT ON THE BASIS OF THE TWO-PARTICLE MODEL}

\section{A. Averaged equations of the motion}

Let us consider the simplest model of the electron bunch, which consists of two particles (pancakes), $i=1$ and 2, placed at a distance $l(t)=z_{1}(t)-z_{2}(t)$. As a first approximation, we consider motion of the particles in the undulator field, which is not perturbed by the Coulomb interaction between the two particles. We introduce the complex transverse velocity, $v_{+}=v_{x}+i v_{y}$. Then, motion Eq. (1) leads to the following equations for the normalized complex transverse momentum $p_{+}=\gamma v_{+} / c$, the normalized axial momentum $p_{z}=\gamma v_{z} / c$, and for the relativistic Lorentz factor $\gamma$ :

$\frac{d p_{+}}{d t}-i \frac{\Omega}{\gamma} p_{+}=-i \frac{e v_{z}}{m c^{2}} B_{u} \exp (i h z), \quad \frac{d p_{z}}{d t}=0, \quad \frac{d \gamma}{d t}=0$.

Here, $\Omega=e B_{0} / m c$ is the nonrelativistic cyclotron frequency corresponding to the axial magnetic field $B_{0}$.

We find the forced solution of Eq. (6), which describes only the undulator oscillations of particles,

$$
p_{+}=-p_{u} \exp (i h z) .
$$

As for the free cyclotron oscillations of particles in the axial magnetic field, their appearance can be avoided by the use of a smooth entry of electrons into the undulator. In this case, the amplitude of transverse oscillations of particles in the undulator field is determined by the following formula [14-16]:

$$
p_{u}=\frac{K}{\Delta},
$$

where $\Delta=1-\Omega / \gamma h v_{z}$ describes the difference between the relativistic cyclotron frequency $\Omega / \gamma$ and the undulator frequency $h v_{z}$, and $K=e B_{u} / m c^{2} h$ is the undulator factor, which coincides with the normalized undulator momentum of electrons, $p_{u}=K$, in the case, when the axial magnetic field is absent $(\Delta=1)$.

In the approximation of the zero Coulomb interaction, the normalized axial electron momentum $p_{z}$ and the relativistic Lorentz factor $\gamma$ stay constant. Naturally, in this case, the axial velocity is also constant, $v_{z, 1}=v_{z, 2}=v_{z 0}$, and the axial size of the bunch $l=z_{1}-z_{2}$ does not change.

The Coulomb interaction leads to a change in both axial velocities and energies of electrons:

$\frac{d z_{i}}{d t}=v_{\mathrm{z}, \mathrm{i}}=v_{\mathrm{z} 0}+c \mu \times\left(\gamma_{i}-\gamma_{0}\right), \quad i=1$ and 2.

Here, $\mu=\frac{1}{c} \frac{d v_{z}}{d \gamma}$ describes the change in the axial electron velocity caused by a change in the relativistic electron 
energy. Therefore, the evolution of the distance between the two particles, $l=z_{1}-z_{2}$, is described by the following equation:

$$
\frac{d l}{d z_{0}}=\frac{c}{v_{z 0}} \mu \times\left(\gamma_{2}-\gamma_{1}\right) \approx \mu \times \delta \gamma
$$

Here, $z_{0}=v_{z 0} t$ is the distance passed by the electron bunch in the case, when the Coulomb interaction is absent, and $\delta \gamma=\gamma_{2}-\gamma_{1}$ is the difference in energies of the two particles. The evolution of energies of the two particles is described by the following equation:

$$
m c^{2} \frac{d \gamma_{i}}{d t}=-e v_{z, i} E_{i} \approx-e v_{z 0} E_{i}, \quad i=1 \text { and } 2 .
$$

Since the Coulomb forces acting between the two macroparticles (pancakes) have the same absolute values,

$$
E_{1}=-E_{2} \approx \frac{-2 \pi Q}{S} \times \operatorname{sign}(l) \times\left[1-\frac{1}{\sqrt{1+R^{2} / \gamma_{0}^{2} l^{2}}}\right],
$$

Eq. (10) leads to the following equation for the difference $\delta \gamma=\gamma_{2}-\gamma_{1}$ in energies of the two particles:

$$
\frac{d \delta \gamma}{d z_{0}}=\frac{\alpha}{l_{0}} \times \operatorname{sign}(l) \times f(l) .
$$

Here, $\alpha=\frac{4 \pi e Q l_{0}}{m c^{2} S}=\frac{4 I}{I_{A}} \frac{l_{0}^{2}}{R^{2}}$ is the normalized electric charge of the electron bunch, and

$$
f(l)=\left[1-\frac{1}{\sqrt{1+R^{2} / \gamma_{0}^{2} l^{2}}}\right]
$$

is a function describing the transverse inhomogeneity of the bunch.

Let us introduce the relative length of the bunch, $L=l / l_{0}$, as well as the normalized axial coordinate $Z=z_{0} / l_{0}$. Then Eqs. (9) and (11) form the following set:

$$
\frac{d L}{d Z}=\mu \times \delta \gamma, \quad \frac{d \delta \gamma}{d Z}=\alpha \times \operatorname{sign}(L) \times f(L),
$$

with the initial conditions $L(0)=1$ and $\delta \gamma(0)=0$.

In Eq. (12), the transverse inhomogeneity of the bunch is described by the following function:

$$
f(L)=\left[1-\frac{1}{\sqrt{1+r^{2} / L^{2}}}\right]
$$

where $r=R / \gamma_{0} l_{0}$ is the normalized radius of the bunch, which represents the ratio between the radius $R$ and the initial length of the bunch in the corelated system, $l_{0}^{\prime}=\gamma_{0} l_{0}$. The case of $r \gg 1$ corresponds to the situation, when the charged pancake can be considered as an infinite charged plane, so that its electric field is independent on the axial coordinate, $f(L) \approx 1$. In the opposite case, $r \ll 1$, the electric field of the pancake decreases with the distance similar to the field of a pointlike particle, $f(L) \approx r^{2} / 2 L^{2}$. In general, function $f(L)$ is limited by the following interval:

$$
0<f(L)<1 \text {. }
$$

Let us find the factor $\mu=\frac{1}{c} \frac{d v_{z}}{d \gamma}$, which can be considered as the inversed effective mass of the particle, $\mu \propto M_{\text {eff }}^{-1}$. We take into account that normalized components of the electron velocity $\beta_{z, u}=v_{z, u} / c=p_{z, u} / \gamma$ are related with the Lorentz factor by the following general relativistic formula:

$$
\beta_{z}^{2}=1-\gamma^{-2}-\beta_{u}^{2}
$$

and the undulator velocity is determined by Eq. (7):

$$
\beta_{u}=\frac{K}{\gamma \Delta}=\frac{K}{\gamma-b / \beta_{z}},
$$

where $b=\Omega / h c \approx \gamma(1-\Delta)$ is the normalized axial magnetic field. Having applied operator $d / d \gamma$ to Eq. (13), one obtains

$$
\beta_{z} \frac{d \beta_{z}}{d \gamma}=\gamma^{-3}+\frac{K^{2}}{\left(\gamma-b / \beta_{z}\right)^{3}} \times\left[1+\frac{b}{\beta_{z}^{2}} \frac{d \beta_{z}}{d \gamma}\right] .
$$

In the case of relativistic electrons $\left(\beta_{z} \rightarrow 1, \gamma \gg 1\right)$ and small enough undulator factor $K$, Eq. (15) leads to the following formula for the factor $\mu$ :

$$
\mu=\frac{d \beta_{z}}{d \gamma} \approx \gamma^{-3}\left[1+\frac{K^{2}}{\Delta^{3}}\right]
$$

\section{B. Negative-mass effect}

According to Eq. (12), the dynamic of the mutual motion of the two particles at the beginning of their interaction $(Z \rightarrow 0)$ is determined by the sign of the factor $\mu$ :

$$
L(Z) \approx 1+\mu \times \alpha \times f(1) \times \frac{Z^{2}}{2} .
$$

In the positive-mass regime $(\mu>0)$, the Coulomb interaction leads to repulsion of the electrons, so that the axial bunch size increases, $L(Z>0)>1$. In contrast, in the negative-mass regime $(\mu<0)$ the axial size decreases $[L(Z>0)<1]$, because in this case the Coulomb interaction leads to attraction of the particles.

Let us describe this effect in detail. The Coulomb interaction leads to an increase in the energies of the particle placed in the bunch front and to a decrease in the 


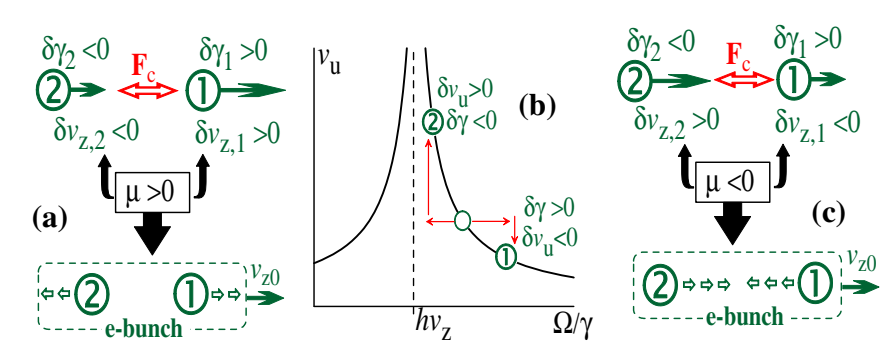

FIG. 3. The model of an electron bunch consisting of two particles. (a) Coulomb repulsion of the two particles in the positive-mass regime. (b) Typical dependence of the oscillatory (undulator) velocity of a particle on the cyclotron frequency. (c) Coulomb attraction of the two particles in the negative-mass regime.

energies of the electron moving in the bunch tail. In the normal, "positive-mass" regime, when $\mu=\frac{1}{c} \frac{d v_{z}}{d \gamma}>0$, the increase/decrease in the electron energy corresponds to the increase/decrease in its axial velocity. In this case, the Coulomb interaction results in repulsion of the particles and, therefore, in degradation of the bunch [Fig. 3(a)].

In the negative-mass regime $\left(\mu=\frac{1}{c} \frac{d v_{z}}{d \gamma}<0\right)$, the increase/ decrease in the electron energy corresponds to the decrease/ increase in its axial velocity. According to Eq. (16), this situation is realized when the relative difference between the relativistic cyclotron frequency and the undulator frequency, $\Delta=1-\Omega / \gamma h v_{z}$, is negative, and its absolute value is small enough:

$$
|\Delta|<K^{2 / 3}, \quad \Delta<0 .
$$

This means that the cyclotron frequency should slightly exceed undulator frequency, $\Omega / \gamma>h v_{z}$ and $\Omega / \gamma \sim h v_{z}$.

The existence of the negative-mass regime is due to the resonance-like dependence of the velocity of transverse undulator oscillations of a particle on the cyclotron frequency [Fig. 3(b)]. Actually, the undulator is a source of a periodic force acting to a particle, which moves in axial magnetic field and, therefore, can be considered as a free cyclotron oscillator. Due to this fact, the amplitude of the forced undulator oscillations is inversely proportional to the difference between the frequencies of forced and free oscillations, $\left|v_{u}\right| \propto\left(h v_{z}-\Omega / \gamma\right)^{-1} \propto \Delta^{-1}$ [see Eq. (14)]. In the negative-mass range of parameters, when $\Omega / \gamma>h v_{z}$, the undulator velocity decreases with an increase in the cyclotron frequency. In this situation, an increase in the energy of a particle moving in the front of the electron bunch shifts the cyclotron frequency closer to the undulator frequency, $\Omega / \gamma \rightarrow h v_{z}$, so that $|\Delta|$ decreases. According to Eqs. (13) and (14), this leads to an increase in its oscillatory velocity and to a decrease in the axial velocity. As for the particle moving in the tail of the bunch, a decrease in its energy due to the Coulomb repulsion leads to an increase in its axial velocity.
Therefore, both particles shift to the center of the electron bunch [Fig. 3(c)]. This effect is proposed earlier $[12,13]$ in a straightforward way for stabilization of the dense electron bunch moving through a relatively long undulator. In this paper, we consider a possibility to use the negative-mass effect to provide axial compression of the electron bunch.

\section{Phase-plane analysis}

Let us analyze the motion of particles on the phase plane $(\delta \gamma, L)$. In the negative-mass regime $(\mu<0)$, Eq. (12) can be rewritten in the following form:

$$
\delta \gamma \times d(\delta \gamma)=\frac{-\alpha}{|\mu|} \times \operatorname{sign}(L) \times f(L) \times d L .
$$

First, we consider the case $r=R / \gamma_{0} l_{0} \gg 1$, when the electron bunch is so short that the Coulomb electric field inside the bunch is independent on the axial coordinate, $f(L) \approx 1$. Then Eq. (18) has the following solution satisfying the condition $\delta \gamma=0$ at $L=1$ :

$$
\delta \gamma= \pm W \times \begin{cases}\sqrt{1-L}, & L>0 \\ \sqrt{1+L}, & L<0\end{cases}
$$

Here, $W=\sqrt{\frac{2 \alpha}{|\mu|}}$. According to formula (19), evolution of the bunch consisting of the two particles is described on the phase plane $(\delta \gamma, L)$ as periodic oscillations (motion along a finite trajectory) of the system. These oscillations correspond to counterphase oscillations of the two particles around the moving center of the bunch. In this process, the variation of the normalized distance between the particles stays within the interval $-1 \leq L \leq 1$, whereas the difference in energies of the particles is changed within the interval $-W \leq \delta \gamma \leq W$ (Fig. 4).

The initial state of the bunch $(L=1$ and $\delta \gamma=0)$ corresponds to the point A on the phase plane (Fig. 4). Attraction of electrons leads to transition to the point $\mathrm{B}$, where electrons have equal axial coordinates $(L=0)$ but different energies. Actually, the first electron (moving in the front) is accelerated by the Coulomb electric field, whereas the second electron (moving in the tail) is decelerated, so that the difference $\delta \gamma=\gamma_{1}-\gamma_{2}=W$ is positive.

The transition from stage A to stage $B$ is the first quarter period of the oscillation of the system on the phase plane. The axial distance passed by the electron beam during this quarter period can be easily found from Eq. (12):

$$
Z_{\mathrm{A} \rightarrow \mathrm{B}}=\sqrt{\frac{2}{|\mu| \alpha}}=\frac{2}{|\mu| W} .
$$

In the process of the second quarter period of the motion along the finite trajectory on the phase plane $(B \rightarrow C)$, the two particles continue their movement without changing direction with respect to the bunch center; namely, the first 


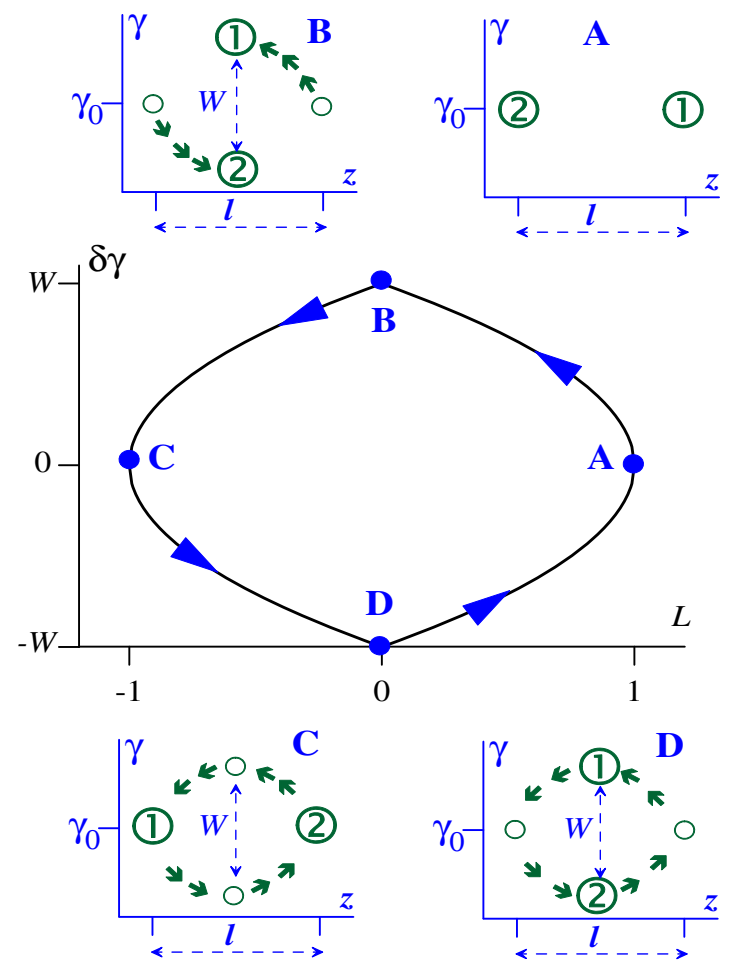

FIG. 4. The model of an electron bunch consisting of two particles. Phase plane describing the evolution of the bunch in the negative-mass undulator, as well as axial positions and energies of the two electrons at different moments of the time, including the initial state (A) and the point of the maximal axial compression (B).

electron goes in the negative direction, whereas the second one goes in the positive direction. As a result, $L=-1$ and $\delta \gamma=0$ in point $\mathrm{C}$. This means that energies of both particles are the same again, the first particle arrives at the initial position of the second particle, and vice versa. After the third and fourth quarter periods $(C \rightarrow D \rightarrow A)$, the system returns to the initial state.

Note again that Eq. (19) described the phase trajectory of the system only in the approximation of an infinitely short electron bunch, $r=R / \gamma_{0} l_{0} \rightarrow \infty 1$. In the general case, Eq. (19) should be transformed as follows:

$\delta \gamma= \pm W \times \begin{cases}{\left[1-L+\sqrt{L^{2}+r^{2}}-\sqrt{1+r^{2}}\right]^{1 / 2},} & L>0 \\ {\left[1+L+\sqrt{L^{2}+r^{2}}-\sqrt{1+r^{2}}\right]^{1 / 2},} & L<0 .\end{cases}$

In this case, evolution of the bunch is described on the phase plane $(\delta \gamma, L)$ as periodic oscillations along finite trajectories, whose forms depend on the factor of the transverse inhomogeneity $r$, but they are quite similar to the phase-plane trajectory in the case of $r \rightarrow \infty$ (Fig. 5). The variation of the normalized distance between the particles stays within the interval $-1 \leq L \leq 1$ independently on the factor $r$. As for the difference in energies of the

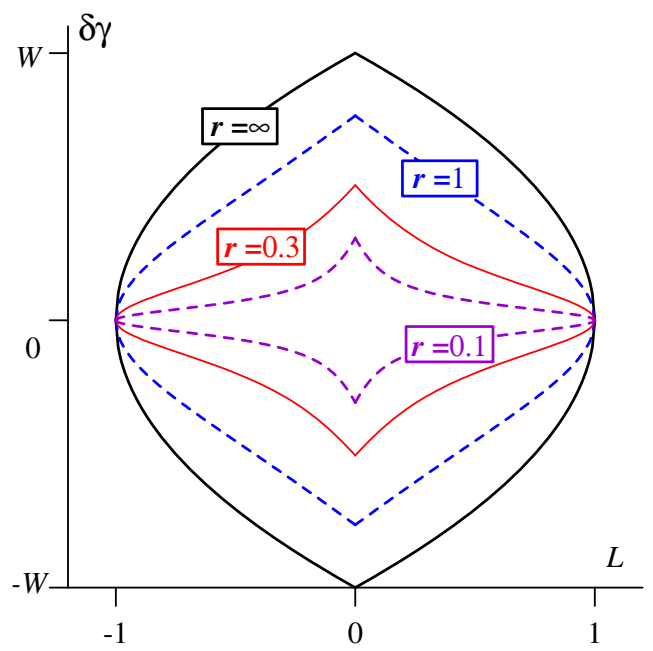

FIG. 5. The model of an electron bunch consisting of two particles. Phase planes describing the evolution of the bunch in the negative-mass undulator at different factors of the transverse inhomogeneity $r$.

two particles, it is changed within the interval $-W_{r} \leq$ $\delta \gamma \leq W_{r}$, where

$W_{r}=W \times F(r), \quad F(r)=\left[1+r-\sqrt{1+r^{2}}\right]^{1 / 2}$.

The amplitude of oscillations of the difference in energies of the two particles decreases with a decrease of $r$ (this corresponds to an enhancement of the transverse inhomogeneity of the electron bunch). This is described by a decrease in the value of function $F(r)$ from $F(\infty)=1$ down to $F(r) \approx \sqrt{r}$ at $r \ll 1$.

\section{Estimation for the compression distance and the energy spread}

Evidently, the optimal compression of the bunch is provided during the first quarter period of the motion of the system along the phase-plane trajectory $A \rightarrow B$ (Fig. 4). In the simplest two-particle model considered above, the length of the electron bunch is compressed in point $\mathrm{B}$ down to zero. Naturally, this statement should be corrected by simulations of behavior of an electron bunch consisting of a more complicated ensemble of electrons. However, the two-electron model can be used to get estimations for the distance required to provide the effective compression of the bunch, as well as for the spread in electron energy encountered in the process of the compression.

The length of the compression region can be estimated from Eq. (20) that needs to be updated taking into account the transverse inhomogeneity:

$$
\frac{z_{\text {comp }}}{l_{0}} \approx \frac{2}{|\mu| W_{r}}=\frac{1}{F(r)} \sqrt{\frac{2}{|\mu| \alpha}} .
$$

This leads to the following estimation for the length of the compressing region: 


$$
z_{\mathrm{comp}} \approx \frac{R}{F(r)} \times \frac{\gamma^{3 / 2}}{\sqrt{\mu_{\Delta}}} \times \sqrt{\frac{I_{A}}{2 I}}
$$

Here, $\mu_{\Delta}=\left|1+\frac{K^{2}}{\Delta^{3}}\right|=\frac{K^{2}}{|\Delta|^{3}}-1$ is the factor taken from Eq. (16); it describes the influence of the proximity of the undulator and cyclotron frequencies on the effective longitudinal mass of electrons. Equation (22) gives the following estimation for the spread in energy encountered in the process of the compression:

$$
\frac{\delta \gamma}{\gamma} \approx F(r) \times \frac{\gamma^{1 / 2}}{\sqrt{\mu_{\Delta}}} \times \frac{l_{0}}{R} \times \sqrt{\frac{8 I}{I_{A}}} .
$$

As a special example, let us study the behavior of estimations (23) and (24) in the situation when the initial bunch length $l_{0}$ increases, whereas the total charge of the bunch $Q \approx I l_{0} / c$ stays constant. In the case of a long electron bunch $\left(r=R / \gamma_{0} l_{0} \ll 1\right)$, the factor of the weakening of the Coulomb field decreases with an increase in the bunch length as follows: $F(r) \approx \sqrt{r} \approx \sqrt{R / \gamma_{0} l_{0}}$. In this situation, the compression length is proportional to the initial length of the bunch, whereas the relative spread in energy encountered in the process of the compression is independent on both the initial $l_{0}$ and the initial electron energy:

$$
z_{\mathrm{comp}} \approx l_{0} \times \gamma^{2} \times \sqrt{\frac{I_{A} R}{2 \mu_{\Delta} Q c}}, \quad \frac{\delta \gamma}{\gamma} \approx \sqrt{\frac{8 Q c}{\mu_{\Delta} I_{A} R}} .
$$

Note that the simplest model considered in this section predicts the zero compression factor $l / l_{0}$ for the "bunch" consisting of two particles, which is achieved at the distance equal to the quarter period of the motion along the phase-plane trajectory determined by Eq. (23). In order to develop this analysis to the case of a multiparticle bunch, one should consider separately the motion of every pair of particles, which are placed initially inside the bunch symmetrically (with respect to the bunch center) and have a distance between themselves $\delta z_{0}<l_{0}$ [Fig. 6(a)]. In this case, one should use in Eq. (11) normalization not to the total length of the bunch $l_{0}$ but to the initial between these two particles $\delta z_{0}$. In addition, the normalized Coulomb electric field acting to these two particles, $\alpha$, is determined not by the total bunch charge $Q$ but by the charge of the section of the bunch between these two particles, $q=Q \delta z_{0} / l_{0} \approx I \delta z_{0} / c$ :

$$
\alpha=\frac{4 \pi e}{m c^{2} S} \times q \delta z_{0}=\frac{4 I}{I_{A}} \frac{\delta z_{0}^{2}}{R^{2}}
$$

This leads to the formula for the quarter period of the motion of these two particles along the phase-plane trajectory (the distance required for the "meeting" of these two particles) that differs from formula (23) the only by factor of transverse inhomogeneity of the bunch, $F$ :

$z_{\text {comp }}\left(\delta z_{0}\right) \approx \frac{R}{F(r)} \times \frac{\gamma^{3 / 2}}{\sqrt{\mu_{\Delta}}} \times \sqrt{\frac{I_{A}}{2 I}}, \quad r=R / \gamma_{0} \delta z_{0}$.

Thus, two particles placed initially inside the bunch meet each other before the pair of particles on the edges of the bunch [Figs. 6(b) and 6(c)]:

$$
\frac{z_{\text {comp }}\left(\delta z_{0}\right)}{z_{\text {comp }}\left(l_{0}\right)}=\frac{F\left(R / \gamma_{0} l_{0}\right)}{F\left(R / \gamma_{0} \delta z_{0}\right)}<1
$$

The relative spread in optimal distances corresponding to different pairs of particles is determined by the function $F$ describing the transverse inhomogeneity,

$$
\frac{z_{\mathrm{comp}}\left(l_{0}\right)-z_{\mathrm{comp}}(0)}{z_{\mathrm{comp}}\left(l_{0}\right)}=1-F\left(R / \gamma_{0} l_{0}\right),
$$

and this spread is one of the factors limiting the minimal possible compression ratio $l / l_{0}$. Evidently, this factor is important, when the initial length of the bunch in the corelated system is comparable with the transverse size of the bunch, $\gamma_{0} l_{0} \sim R$; at long bunch lengths $\left(\gamma_{0} l_{0} \gg R\right)$ this spread limits to $100 \%$ :
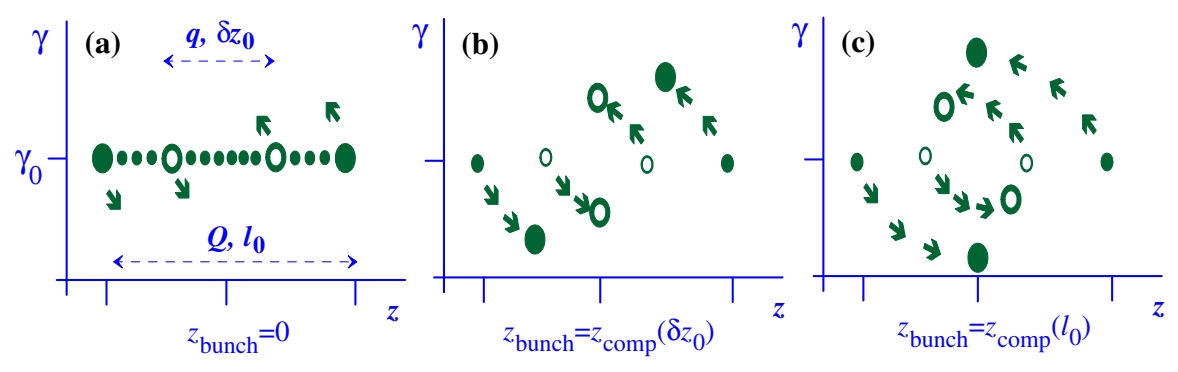

FIG. 6. Different motion of two pairs of electrons, namely, the pair of particles initially placed on the edges of the bunch and a pair of electrons initially placed inside the bunch: (a) initial state, (b) the "meeting point" of the inner pair, and (c) the meeting point of the two edge particles. 


$$
\frac{z_{\text {comp }}\left(l_{0}\right)-z_{\text {comp }}(0)}{z_{\text {comp }}\left(l_{0}\right)} \approx 1-\sqrt{R / \gamma_{0} l_{0}} .
$$

One more factor limiting the minimal possible compression ratio $l / l_{0}$ is not taken into account in this simplest model; this is initial spread in electron velocity. Evidently, the importance of this factor increases with the increase in the axial distance required to achieve the effective compression of the bunch. According to estimations (25), if the bunch charge is fixed, then the compression distance is proportional to the initial length of the bunch, $z_{\text {comp }} \propto l_{0}$. Therefore, this factor should also lead to a deterioration of the compression process with increasing initial bunch length.

\section{NUMERICAL SIMULATION OF DYNAMICS OF A SHORT ELECTRON BUNCH IN THE NEGATIVE-MASS UNDULATOR}

\section{A. Parameters of simulated system}

The proposed regime of the compression of electron bunches has been numerically simulated on the basis of Eqs. (1)-(3). We have studied the motion of the electron bunch with the parameters close to those discussed for the Israeli $\mathrm{THz}$ Source [13]: an initial charge of $0.3 \mathrm{nC}$, diameter $1 \mathrm{~mm}$, an initial length of $0.1-1.0 \mathrm{~mm}$, and the Lorentz factor $\gamma=12$ in the helical undulator with a period of $2.5 \mathrm{~cm}$ and the undulator parameter $K=0.5$.

We assume that at the beginning of the compression process, electrons are distributed uniformly the length of the bunch, $0 \leq z_{0} \leq l_{0}$, and there is a spread in initial electron energy, $\delta \gamma_{0} / \gamma_{0}=0.01$. It is assumed that the initial state of every electron is its oscillation in the undulator field unperturbed by the Coulomb interaction, which is determined by the forced solution of Eq. (6):

$p_{+}(t=0)=-p_{u} \exp \left(i h z_{0}\right), \quad p_{u}=\frac{K}{\Delta}, \quad \Delta=1-\Omega / \gamma_{0} h v_{z 0}$.

This corresponds to the situation when the electron bunch enters into the undulator via an entry region, where the undulator factor smoothly increases from zero up to the nominal value, and the Coulomb interaction is neglected inside this region. The smooth entry of electrons into the undulator ensures the absence of the free cyclotron oscillations of particles in the regular undulator.

Note that the existence of the nonregular entry region leads also to an additional axial compression of the bunch. This is due to the fact that if the undulator field increases with the coordinate, then the bunch head is wiggled by a stronger undulator field (as compared to the bunch tail), and, therefore, it has smaller axial velocity, so that the tail "catches up" with the head. Naturally, this is a useful effect, but we have excluded it from consideration in this article.

\section{B. Axial compression of the electron bunch}

In order to characterize the axial compression of the bunch in simulations, we introduce the following compression factor:

$$
l(t) / l_{0}=\frac{D[z(t)]}{D[z(0)]} .
$$

Here,

$$
D[z(t)]=\sqrt{\frac{1}{0.9 N} \sum_{j}\left[z_{j}(t)-\langle z(t)\rangle\right]^{2}}
$$

is the dispersion of axial positions of electrons. In this formula,

$$
\langle z(t)\rangle=\frac{1}{0.9 N} \sum_{j} z_{j}(t)
$$

is the averaged current coordinate of the bunch, $N$ is the number of particles in the bunch, and the summation is made over $90 \%$ of electrons, so that we have excluded from the consideration $10 \%$ of the "lost" particles $(5 \%$ with largest axial coordinates $z_{j}$ and $5 \%$ with smallest coordinates).

Figure 7 illustrates results of simulations for electron bunches with different initial lengths, $l_{0}=0.1 \mathrm{~mm}$, $0.5 \mathrm{~mm}$ and $1 \mathrm{~mm}$. Figure 7(a) shows dependencies of the minimal compression factors $l / l_{0}$ versus the normalized difference between undulator and cyclotron frequencies, $\Delta=1-\Omega / \gamma_{0} h v_{z 0}$. The length of the compressing undulator required to achieve these compression factors is shown in Fig. 7(b). Note that in the negative-mass regime $\Delta$ is negative, and its absolute value is a characteristic of the value of the axial magnetic field used in this system,

$$
B_{0}=B_{\text {res }} \times(1+|\Delta|),
$$

where $B_{\text {res }}$ is the magnitude of the guiding magnetic field corresponding to the exact resonance (to the equality of the two frequencies, $\Delta=0$ ). In the case of parameters considered in these simulations, $B_{\text {res }} \approx 5 \mathrm{~T}$.

According to simulations, the optimal normalized difference between undulator and cyclotron frequencies is $|\Delta|=0.3-0.4$. At bigger differences the negative-mass effect becomes weaker, and at $|\Delta|>K^{2 / 3} \approx 0.62$ [see Eq. (16)] the effective electron mass becomes positive $(\mu>0)$, so that the negative-mass Coulomb attraction is changed to the positive-mass Coulomb repulsion. At small $|\Delta|$ the effective mass becomes too small $(\mu \rightarrow-\infty)$, and the strong negative-mass Coulomb attraction of electrons leads to a significant change in their energies. In this situation, the simplified model considered in Sec. III does not describe the behavior of the system, because the Coulomb perturbations of electron motion in the undulator field cease to be small. 

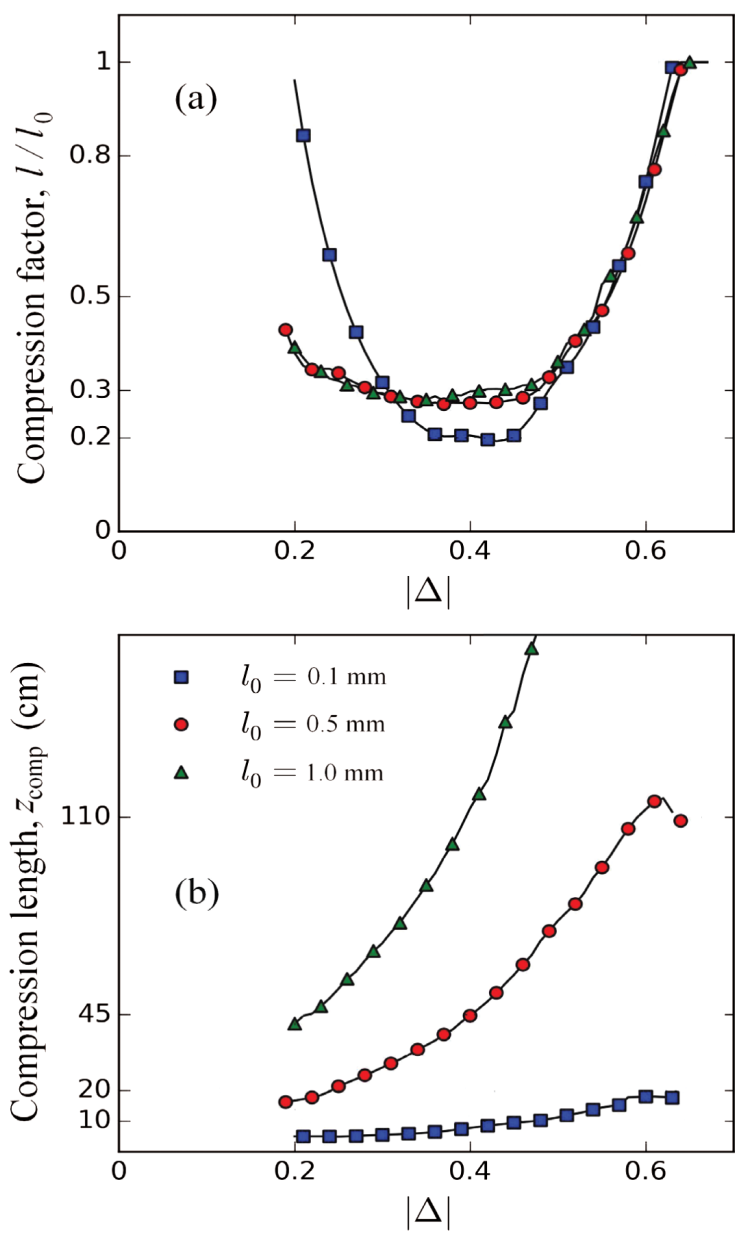

FIG. 7. Minimal compression factors $l / l_{0}$ (a) and lengths of the compressing undulator (b) versus the normalized difference between undulator and cyclotron frequencies, $\Delta$, for electron bunches with initial lengths, $l_{0}=0.01,0.5$ and $1 \mathrm{~mm}$.

Figures 8 and 9 illustrate the dynamic of electron bunches with initial lengths 0.1 and $1.0 \mathrm{~mm}$, respectively. At the input (point 1), electrons are distributed uniformly over the initial length, $0 \leq z_{0, \mathrm{j}} \leq l_{0}$, a spread in initial electron energy, $\delta \gamma_{0} / \gamma_{0}=0.01$, is introduced. At the initial stage of the motion (point 2), the Coulomb attraction of electrons leads to a decrease in the axial size of the bunch. In point 3 of the maximal compression, the effective bunch lengths in both cases are reduced down to a value of $l / l_{0} \approx 0.3$. Note that the initial points 1 and the maximal compression points 3 in Figs. 8 and 9 correspond to points $\mathrm{A}$ and $\mathrm{B}$ on the phase plane shown in Fig. 4.

The simulation results are in good accordance with estimations for the length of the compressing region and for the spread in energy encountered in the process of the compression [formulas (23) and (24)]. In the case of $0.3 \mathrm{nC}$ electron bunch, $I=1 \mathrm{kA}$ for $l_{0}=0.1 \mathrm{~mm}$ and $I=100 \mathrm{~A}$ for $l_{0}=1 \mathrm{~mm}$. The undulator parameter $K=0.5$ and the normalized mismatch between the undulator and cyclotron frequencies $\Delta=-0.4$ result in $\mu_{\Delta} \approx 3$. In the case of the initial bunch length $l_{0}=0.1 \mathrm{~mm}$, the factor of the transverse inhomogeneity is $r=R / \gamma_{0} l_{0} \approx 0.4$, so that according to Eq. (22) $F(r) \approx 0.6$. This leads to the following estimations:

$$
z_{\text {comp }} \approx 6 \mathrm{~cm}, \quad \frac{\delta \gamma}{\gamma} \approx 0.16
$$

As for the case of a 10 times longer bunch $\left(l_{0}=1 \mathrm{~mm}\right)$, the factor of the transverse inhomogeneity is 10 times smaller, $r=R / \gamma_{0} l_{0} \approx 0.04$, and the factor of the weakening of the Coulomb field is $F(r) \approx \sqrt{r} \approx 0.2$. In this situation, the compression length and the spread in energy in the point of the maximal compression are estimated as follows:

$$
z_{\text {comp }} \approx 55 \mathrm{~cm}, \quad \frac{\delta \gamma}{\gamma} \approx 0.17
$$

\section{Long-length dynamics of the electron bunch in the negative-mass undulator}

After the point of the maximal compression, a decompression of the bunch takes place (points 4 and 5 in Figs. 8 and 9). In principle, this corresponds to the motion of the system along the segment B-C of the trajectory of the phase plane shown in Fig. 4(a) due to the inertia of the particles. However, numerical simulations performed within the framework of the "multiparticle" model of the electron bunch show a more complicated (as compared to the "twoparticle" model illustrated by the phase plane shown in Fig. 4) behavior of the bunch after the point of the maximal compression.

Figure 10 illustrates three different examples of dynamics of the motion of a bunch with a relatively long initial length $\left(l_{0}=1 \mathrm{~mm}\right)$ through the negative-mass undulator. These three plots show dependencies of axial positions of different particles with respect to the averaged center of the bunch, $z_{\mathrm{j}}-\left\langle z_{\mathrm{j}}\right\rangle$, versus the current axial coordinate, $z$. These three examples differ only in the mismatch between the undulator and cyclotron frequencies, $\Delta$. Note that the absolute value of $\Delta$ determines the effective strength of the Coulomb interaction (attraction) of electrons. According to Eq. (16), a decrease in $|\Delta|$ leads to an increase in the factor $\mu$; this results in a stronger negative-mass Coulomb interaction between electrons.

Figure 10(a) illustrates the case of $\Delta=-0.55$, when the negative-mass Coulomb interaction between electrons is relatively small. Although a small fraction of particles leaves the bunch after passing the point of the maximal compression, the main part of the bunch stays stable, such that electrons oscillate around the averaged center of the bunch in the process of the motion of the bunch through the undulator. Note that these oscillations are well described by the simplest two-particle model considered in Sec. III; they correspond to the motions of electrons along finite trajectories on the phase planes shown in Figs. 4 and 5. 

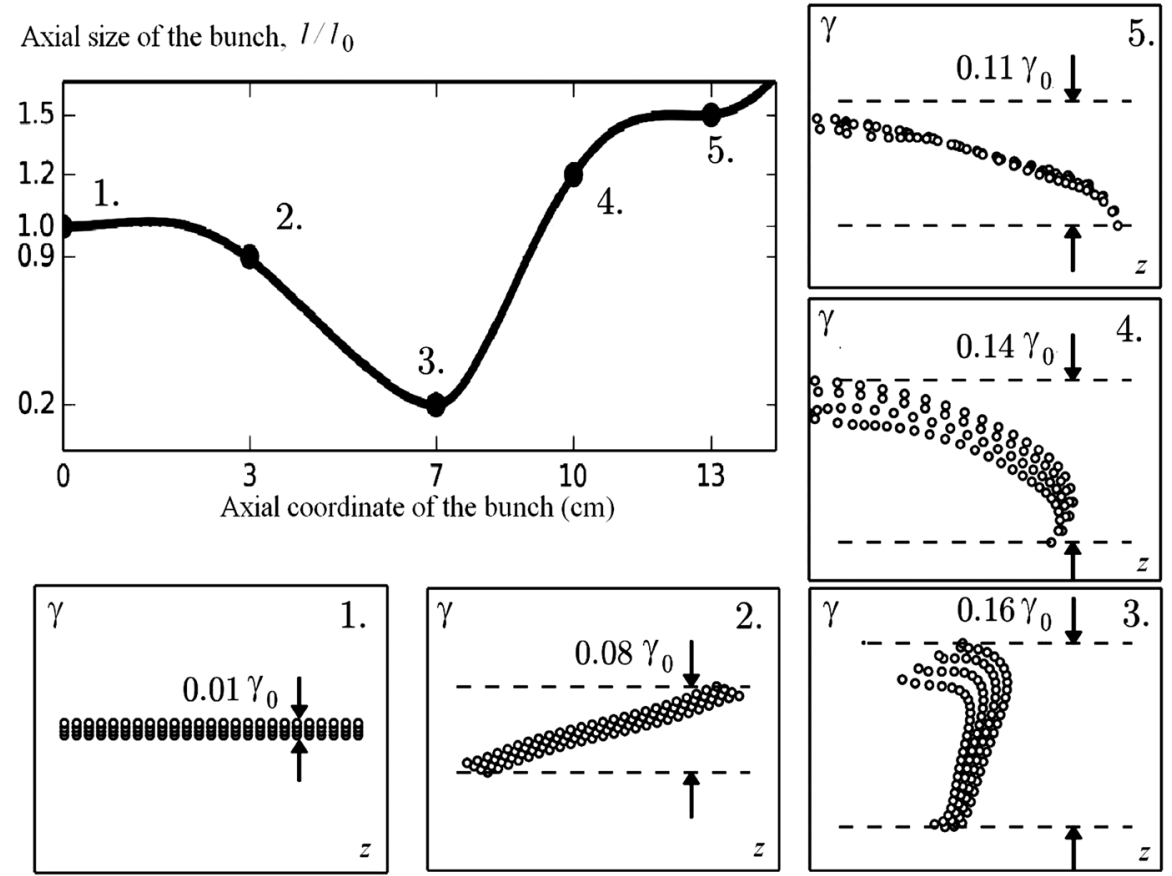

FIG. 8. Dynamics of the electron bunch with an initial length of $0.1 \mathrm{~mm}$ moving through a negative-mass undulator $(\Delta=-0.4)$. Effective bunch length normalized to the initial value, $l / l_{0}$, versus the axial coordinate of the bunch, as well as distributions of electrons on the phase planes (energy/axial position) at different stages of the bunch motion.

However, at a stronger Coulomb interaction between electron, the simplest two-particle model developed in Sec. III does not describe the behavior of the electron bunch after the point of the maximal compression. This is true especially in the case of a relatively long bunch, which is illustrated by Figs. 9 and 10. It is shown in Fig. 9 that after point 3 of the maximal compression, electrons form two separated quasistable bunches of attracting particles
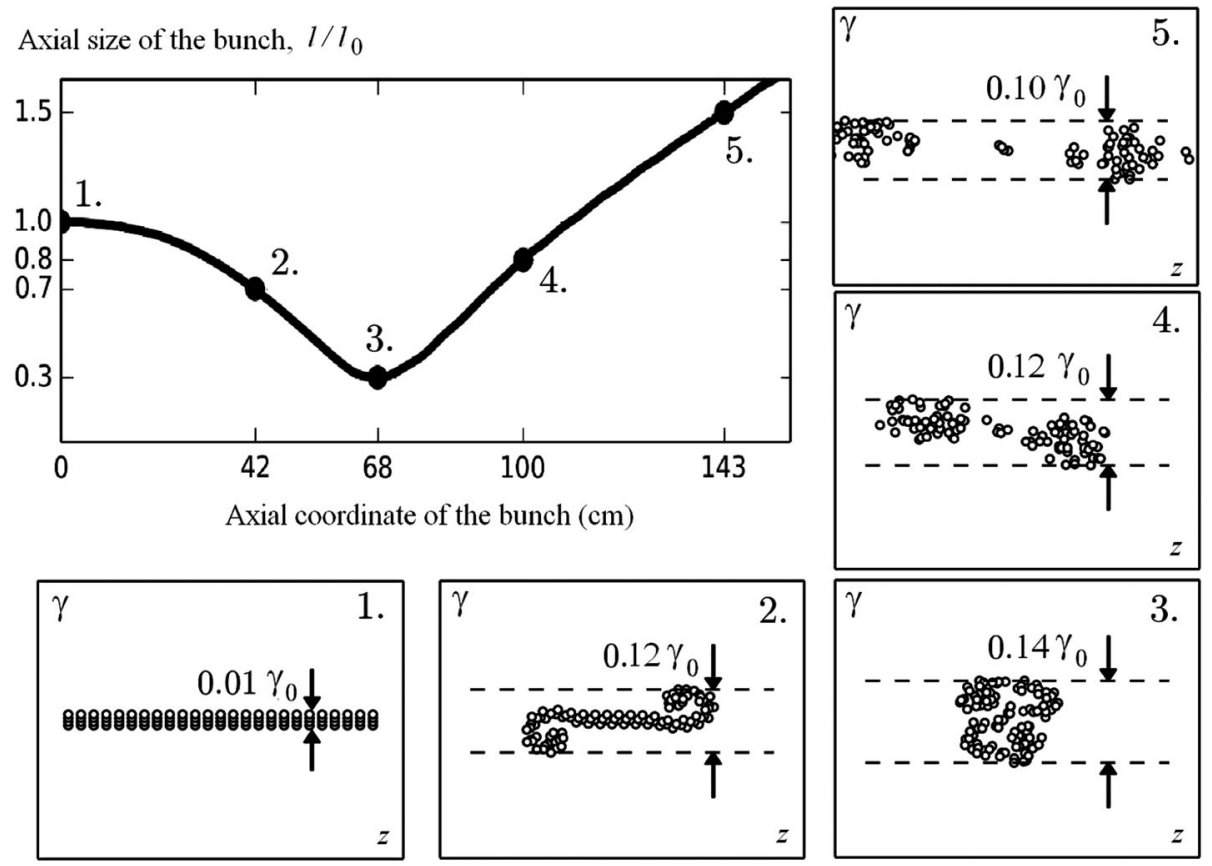

FIG. 9. Dynamics of the electron bunch with an initial length of $1 \mathrm{~mm}$ moving through a negative-mass undulator $(\Delta=-0.4)$. Effective bunch length normalized to the initial value, $l / l_{0}$, versus the axial coordinate of the bunch, as well as distributions of electrons on the phase planes (energy/axial position) at different stages of the bunch motion. 


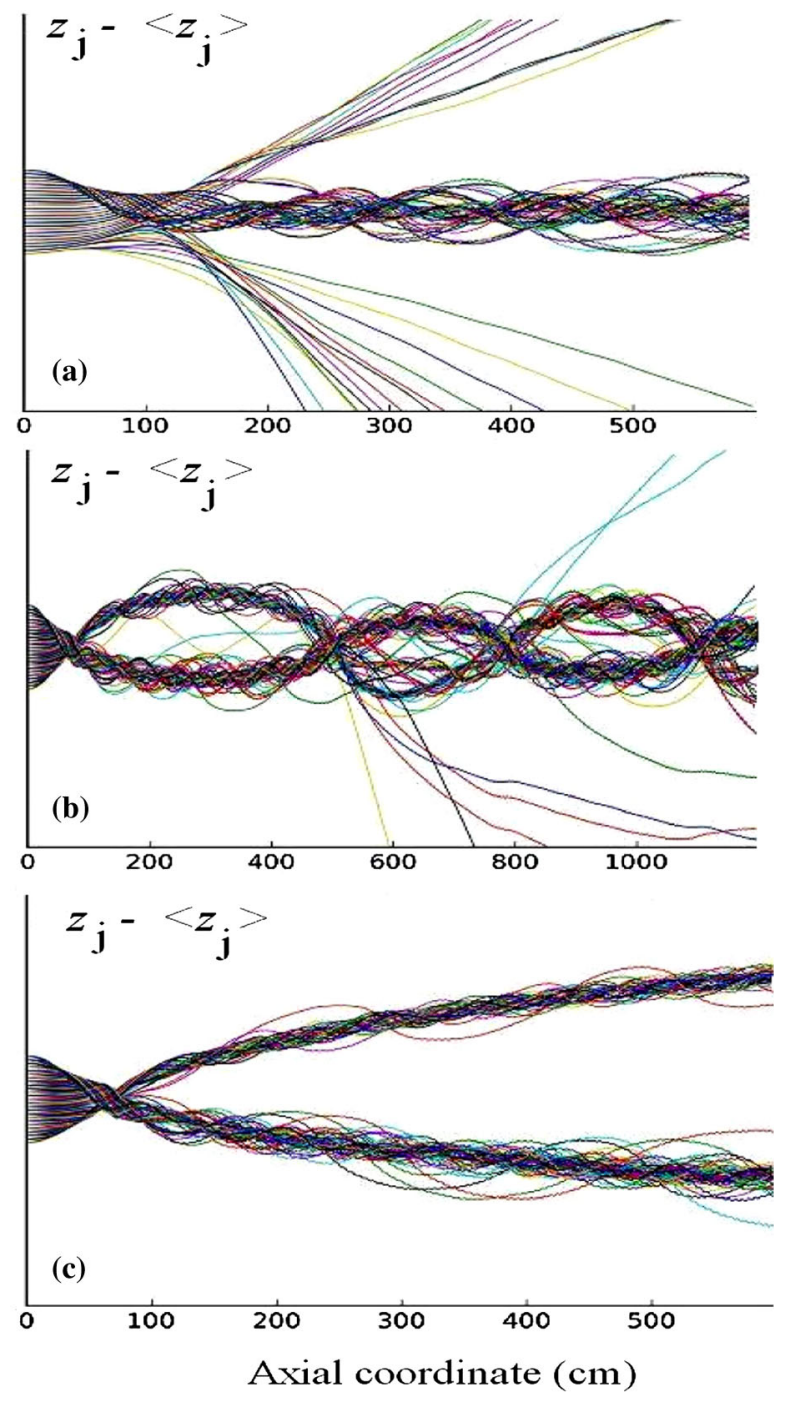

FIG. 10. Long-length dynamic of the electron bunch with an initial length of $1 \mathrm{~mm}$ moving through a negative-mass undulator at various mismatches between undulator and cyclotron frequencies, (a) $\Delta=-0.55$, (b) $\Delta=-0.48$, and (c) $\Delta=-0.46$. Axial positions of different particles with respect to the averaged center of the bunch, $z_{\mathrm{j}}-\left\langle z_{\mathrm{j}}\right\rangle$, versus the current axial coordinate.

(points 4 and 5 in Fig. 9). Further behavior of these two "sub-bunches" is determined by the distance between them acquired after passage of the point of the maximal compression. If the effective Coulomb interaction is not too strong (the effective mass of the particles is not too small), then the behavior of these two sub-bunches is like a "double planet"; namely, the sub-bunches move together and oscillate around the common center due to their mutual attraction [Fig. 10(b), $\Delta=-0.48$ ]. At even smaller (in absolute value) mismatches [Fig. 10(c), $\Delta=-0.46$ ], the strong Coulomb interaction results in a bigger distance between the sub-bunches, as well as in a significant difference between averaged energies of particles in the sub-bunches. In this situation, the sub-bunches are not attracted. They stay quasistable and gradually move away from each other, propagating along the negative-mass undulator separately with slightly different averaged axial velocities.

\section{CONCLUSION}

Short dense electron bunches produced from laser-driven photoinjectors can be directly exploited for radiation in the $\mathrm{THz}$ frequency range in the regime of spontaneous coherent radiation. This type of radiation is realized when the effective axial length of bunches is shorter than the radiation wavelength. In this situation, there are problems of formation of dense bunches with short enough length, as well as stabilization of the axial size of the bunch during its motion through the radiation region of the $\mathrm{THz}$ source.

In this paper we show that the negative-mass undulator (which was originally proposed to provide the bunch stabilization in the process of the radiation) can be interesting also from the point of view of manipulations with short dense electron bunches, including compression of the axial size of such a bunch. In this work, we describe dynamics of a bunch moving in the negative-mass undulator on the basis of a simplified 1D model, where the bunch is represented as a set of thin pancakes with the stable transverse size. According to simulations, the axial compression factors achieved in the simplest uniform undulator can be as high as 3-5. In simulations we observe also an interesting effect of a complicated dynamics of a relatively long electron bunch, namely, splitting a long bunch into two separated quasistable sub-bunches. This is an important effect limiting the compression of dense electron bunches. On the other hand, this effect can be used in more complicated regimes of the e-bunch manipulation.

The main advantage of the proposed compression scheme is the possibility for its natural use as a first section of a $\mathrm{THz}$ source based on spontaneous coherent emission from short bunches moving through a negative-mass undulator (see Fig. 1). However, the negative-mass compression of electron bunches may be of value in itself. In principle, the well-developed methods of compression of photoinjector bunches based on the energy chirping allow producing bunches with as short lengths as tens fs $\left(\sim 10^{-2} \mathrm{~mm}\right)$ [22-25]. However, they are successful only when the Coulomb forces inside the bunch are not so important. Such a situation takes place either in bunches with typical "photoinjector" electron energies (several $\mathrm{MeV}$ ) and with relatively small charges (at the pC level) [22], or in bunches accelerated up to a high electron energy (hundreds $\mathrm{MeV}$ of even higher) [23-25]. In this paper, we study bunches with relatively low energies $(\sim 5 \mathrm{MeV})$ and the $\mathrm{nC}$ level of the total charge. In this case, the Coulomb forces are the key problem, as they are so strong that they lead to a significant destruction of the bunch during its trip through a distance of the order of tens $\mathrm{cm}$. The proposed negative-mass method solves this problem by means of the change of "sign" of these forces. Evidently, this method can 
be improved by the use of additional techniques (such as energy chirping), but this could be the subject of future works.

\section{ACKNOWLEDGMENTS}

Please, correct the Foundation identification as follow: This work was supported by IAP RAS Projects No. 00352014-0012, and, in part, by the Russian Foundation for Basic Research, Project No. 16-02-00794 A.

[1] J. G. Power, Overview of photoinjectors, AIP Conf. Proc. 1299, 20 (2010).

[2] A. Bartnik, C. Gulliford, I. Bazarov, L. Cultrera, and B. Dunham, Operational experience with nanocoulomb bunch charges in the Cornell photoinjector, Phys. Rev. ST Accel. Beams 18, 083401 (2015).

[3] F. Stephan, C. H. Boulware, M. Krasilnikov, J. Bahr, G. Asova, A. Donat, U. Gensch, H. J. Grabosch, M. Hanel, L. Hakobyan et al., Detailed characterization of electron sources yielding first demonstration of European x-ray free-electron laser beam quality, Phys. Rev. ST Accel. Beams 13, 020704 (2010).

[4] J. B. Rosenzweig, A. Valloni, D. Alesini, G. Andonian, N. Bernard, L. Faillace, L. Ficcadenti, A. Fukusawa, B. Hidding, M. Migliorati, A. Mostacci, P. Musumeci, B. O. Shea, L. Palumbo, B. Spataro, and A. Yakub, Design and applications of an X-band hybrid photoinjector, Nucl. Instrum. Methods Phys. Res., Sect. A 657, 107 (2011).

[5] K. J. P. Quintero, S. Antipov, A. V. Sumant, C. Jing, and S. V. Baryshev, High quantum efficiency ultrananocrystalline diamond photocathode for photoinjector applications, Appl. Phys. Lett. 105, 123103 (2014).

[6] A. Doria, R. Bartolini, J. Feinstein, G. P. Gallerano, and R. H. Pantell, Coherent emission and gain from a bunched electron beam, IEEE J. Quantum Electron. 29, 1428 (1993).

[7] A. Gover, F. V. Hartemann, G. P. Le Sage, N. C. Luhmann, Jr., R. S. Zhang, and C. Pellegrini, Time and Frequency Domain Analysis of Superradiant Coherent Synchrotron Radiation in a Waveguide Free-Electron Laser, Phys. Rev. Lett. 72, 1192 (1994).

[8] Y. Lurie and Y. Pinhasi, Enhanced superradiance from energy-modulated short electron bunch free-electron lasers, Phys. Rev. ST Accel. Beams 10, 080703 (2007).

[9] Y. Lurie, A. Friedman, and Y. Pinhasi, Single pass, THz spectral range free-electron laser driven by a photocathode hybrid rf linear accelerator, Phys. Rev. ST Accel. Beams 18, 070701 (2015).

[10] V. L. Bratman, D. A. Jaroszynsky, S. V. Samsonov, and A. V. Savilov, Generation of ultrashort quasiunipolar electromagnetic pulses from quasiplanar electron bunches, Nucl. Instrum. Methods Phys. Res., Sect. A 475, 436 (2001).

[11] K. Lee, J. Mun, S. H. Park, R.-H. Jang, Y. U. Jeong, and N. A. Vinokurov, Numerical investigation of the radiation characteristics of a variable-period helical undulator, Nucl. Instrum. Methods Phys. Res., Sect. A 776, 27 (2015).

[12] N. Balal, I. V. Bandurkin, V. L. Bratman, E. Magory, and A. V. Savilov. Negative-mass mitigation of Coulomb repulsion for terahertz undulator radiation of electron bunches, Appl. Phys. Lett. 107, 163505 (2015).

[13] Y. Lurie, V. L. Bratman, and A. V. Savilov, Energy enhancement and spectrum narrowing in terahertz electron sources due to negative mass instability, Phys. Rev. Accel. Beams 19, 050704 (2016).

[14] N. S. Ginzburg and N. Yu. Peskov, Nonlinear theory of relativistic ubitrons with electron beams formed in an adiabatically increasing undulator field and a uniform longitudinal magnetic field, Tech. Phys. 58, 859 (1988).

[15] H. P. Freund and T. M. Antonsen, Principles of FreeElectron Lasers (Chapman and Hall, London, 1996).

[16] I. V. Bandurkin, S. V. Kuzikov, and A. V. Savilov, Cyclotron-undulator cooling of a free-electron-laser beam, Appl. Phys. Lett. 105, 073503 (2014).

[17] V. L. Bratman, Instability of orbital motion in a layer of electrons rotating in a uniform magnetic field, Tech. Phys. 46, 2030 (1976).

[18] A. Bondeson and T. M. Antonsen, Space-charge instabilities in gyrotron beams, Int. J. Electron. 61, 855 (1986).

[19] V. L. Bratman and A. V. Savilov, Phase mixing of bunches and decrease of negative- mass instability increments in cyclotron resonance masers, Phys. Plasmas 2, 557 (1995).

[20] A. V. Savilov, Negative-mass instability in magnetroninjection guns, Phys. Plasmas 4, 2276 (1997).

[21] V. L. Bratman, O. Dumbrajs, P. Nikkola, and A. V. Savilov, Space charge effects as a source of electron energy spread and efficiency degradation in gyrotrons, IEEE Trans. Plasma Sci. 28, 633 (2000).

[22] X. H. Lu, C. X. Tang, R. K. Li, H. To, G. Andonian, and P. Musumeci, Generation and measurement of velocity bunched ultrashort bunch of pC charge, Phys. Rev. ST Accel. Beams 18, 032802 (2015).

[23] K. Sakaue, Y. Koshiba, M. Mizugaki, M. Washio, T. Takatomi, J. Urakawa, and R. Kuroda, Ultrashort electron bunch generation by an energy chirping cell attached rf gun, Phys. Rev. ST Accel. Beams 17, 023401 (2014).

[24] M. Schreck and P. Wesolowski, Analytical bunch compression studies for a linac-based electron accelerator, Phys. Rev. ST Accel. Beams 18, 100101 (2015).

[25] J. Zhu, R. W. Assmann, M. Dohlus, U. Dorda, and B. Marchetti, Sub-fs electron bunch generation with sub-10-fs bunch arrival-time jitter via bunch slicing in a magnetic chicane, Phys. Rev. Accel. Beams 19, 054401 (2016). 